\title{
Parameters Optimization for Extended-range Electric Vehicle Based on Improved Chaotic Particle Swarm Optimization
}

\author{
Yongchen Jiang, Cheng Lin and Wanke Cao * \\ Collaborative Innovation Center for Electric Vehicles, Beijing Institute of \\ Technology, Beijing 100081, China \\ *caowanke@bit.edu.cn
}

\begin{abstract}
Extended-range electric vehicle is considered to be the ideal transition type for electric vehicle. The optimal operation curve control strategy was proposed for a 12 meter-long range extended electric bus. With exponential function inertia weight adjustment and local chaos substitution, an improved chaotic particle swarm optimization algorithm was applied to optimize the key parameters of energy management strategy. Based on MATLAB/Simulink, full vehicle model and corresponding control strategy were built. The simulation results with typical city driving cycles illustrate that, comparing with standard particle swarm optimization, the new algorithm can greatly improve the convergence speed and optimizing precision, and the optimal parameters can be obtained.
\end{abstract}

Keywords: Extended-range electric vehicle, Energy management, Simulation, Particle swarm algorithm.

\section{Introduction}

Extended-range electric vehicle (E-REV) can overcome the short driving range and long charging time of a pure electric vehicle, and its structure is relatively simple. Therefore, it is considered to be the ideal transition type for electric vehicle [1].

Energy management control strategy's design and development are the key technology of E-REV. At present, HEV's energy management control strategies mainly focus on the optimization-based strategy, including instantaneous optimization strategy and global optimization strategy [2]. However, in terms of practical engineering application, the rulebased energy management control strategies are more clear and easy to be implemented and developed. Some optimization algorithms are used to optimize the key parameters of E-REV energy management strategy, such as Particle swarm optimization (PSO) and genetic algorithm (GA) optimization, which could effectively improve vehicle performance [3-4].

Since Standard particle swarm optimization (SPSO) has slow convergence rate and was easily trapped in local optimum, an improved chaotic particle swarm optimization (ICPSO) was presented. By means of introducing chaotic mutation, using inertia weight adjustment strategy, velocity and position selection strategy for particles beyond the boundaries, ICPSO can improve the convergence accuracy and convergence rate of SPSO. In this paper, combining with the characteristics of the dynamic system of EREV, ICPSO is applied to optimize the parameters of E-REV energy management control strategy.

Wanke Cao is the corresponding author. 


\section{E-REV Energy Management Control Strategy}

\subsection{Power-Train System Structure}

At present, most extended-range electric vehicles are refitted based on the traditional vehicles, and the E-REV power-train system structure shown in Figure 1. Which includes battery, drive motor, the engine-generator unit (APU), gear box and drive wheels, etc. As the primary power source, the battery is directly connected to the system bus, which meets the desired vehicle dynamic performance and provides pure electric driving range. APU and battery are in parallel connection on the DC bus, which provides drive motor with auxiliary power and charges the battery to increase the vehicle driving range. The power output of drive motor through the main reducer and differential to drive wheels [6].

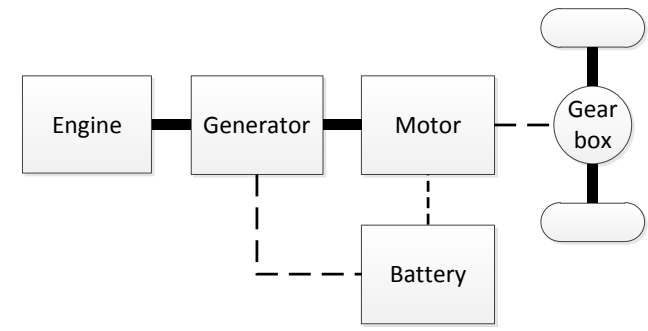

\section{Figure 1. E-REV Power-Train System Structure}

The study reported by Society of Automotive Engineers (SAE) showed that the biggest difference between E-REV and P-HEV is: when the E-REV's batteries energy is sufficient, the batteries and drive motor can meet all the desired vehicle dynamic performance, the engine can be started directly, and switch to extended-range mode instantly [7]. Thus, the energy and power requirement of E-REV's batteries and drive motor is rather high with a large size. And the driving power of the engine only needs to satisfy the power demand of particular conditions under extended-range mode. As a result, low power and small size can be selected.

\subsection{Optimal Operation Curve Control Strategy}

Based on E-REV operation mode, the study of its energy management control strategy is accordingly divided into two stages: pure electric stage and extended-range stage. The control of the former is relatively simple, which main goal is to research and control the vehicle required torque. For the extended-range stage, we should control the power allocation of APU and batteries to reduce fuel consumption and obtain good fuel economy. Since APU is mechanically independent of the drive shaft, the rotational speed and output torque of engine is irrelevant to the drive motor. That is to say, the engine can work in any torque-speed plane.

The optimal operation curve control strategy is based on power-following. According to the battery SOC and the power demand (PL), several different intervals were divided. Each interval has a rule, and each rule corresponds to the specified speed and power of engine. When engine starts, it works on the Engine optimal operation curve which is calculated according to the engine fuel consumption characteristics map and the efficiency map of generator, as shown in Figure 2. 


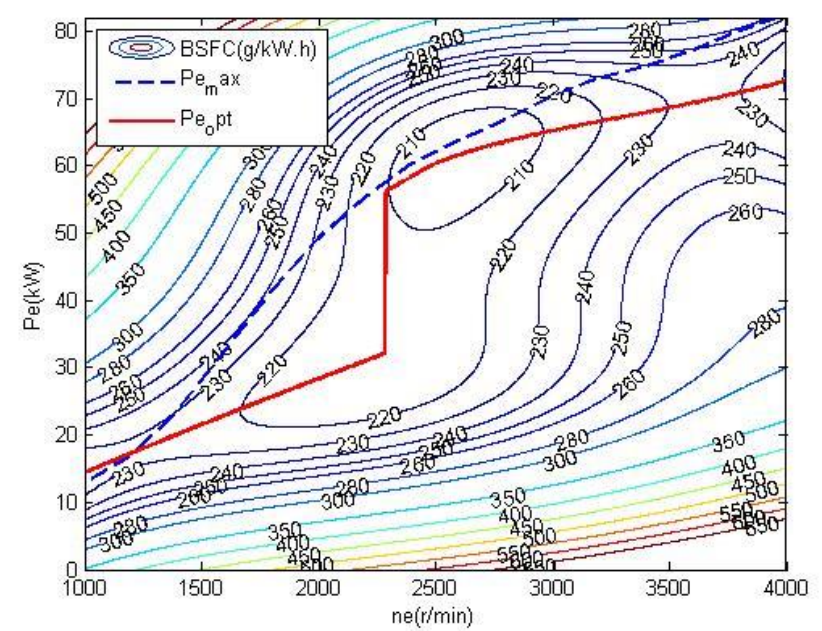

Figure 2. The Optimal Operation Curve of Engine

The logic rules of optimal operation curve control strategy are shown as in Tab.1. Where $S O C_{\text {low }} 、 S O C_{\text {high }}$ are the threshold of battery charging and discharging respectively; $\mathrm{Pe}_{\text {low }} 、 \mathrm{Pe}_{\text {high }}$ are the power demand threshold of engine turning on/off respectively.

Table 1. The Logic Rules of Optimal Operation Curve Control Strategy

\begin{tabular}{|c|c|c|c|}
\hline SOC & SOC $<\mathrm{SOC}_{\text {low }}$ & $\mathrm{SOC}_{\text {low }} \leq \mathrm{SOC}_{L}<\mathrm{SOC}_{\text {high }}$ & $\mathrm{SOC}>\mathrm{SOC}_{\text {high }}$ \\
\hline $0<\mathrm{P}_{L} \leq \mathrm{Pe}_{\text {low }}$ & On, $\mathrm{Pe}=\mathrm{Pe}_{\text {low }}$ & Hold, $\mathrm{Pe}=\mathrm{Pe}_{\text {low }}$ or $\mathrm{Pe}=0$ & Off, $\mathrm{Pe}=0$ \\
\hline $\mathrm{Pe}_{\text {low }}<\mathrm{P}_{L} \leq \mathrm{Pe}_{\text {high }}$ & On, $\mathrm{Pe}=\mathrm{P}_{L}$ & Hold, $\mathrm{Pe}=\mathrm{P}_{L}$ or $\mathrm{Pe}=0$ & Hold, $\mathrm{Pe}=\mathrm{P}_{L}$ \\
\hline $\mathrm{P}_{L}>\mathrm{Pe}_{\text {high }}$ & On, $\mathrm{Pe}=\mathrm{P}_{L}$ & Hold, $\mathrm{Pe}=\mathrm{P}_{L}$ or $\mathrm{Pe}=0$ & On, $\mathrm{Pe}=\mathrm{P}_{L}$ \\
\hline $\mathrm{P}_{L}<0$ & \multicolumn{3}{|c|}{ Off, $\mathrm{Pe}=0$} \\
\hline
\end{tabular}

In this paper, five parameters of the energy control strategy are selected as optimization variables, as shown in Tab. 2. Where, $\square \mathrm{t}$ is the minimum duration of the engine start-stop interval. (The maximum output power of engine is $82 \mathrm{kw}$ )

Table 2. The Optimization Variables of Optimal Operation Curve Control Strategy

\begin{tabular}{|c|c|c|}
\hline Optimization variables & Lower limit & Upper limit \\
\hline$S O C_{\text {low }}$ & 0.2 & 0.4 \\
\hline$S O C_{\text {high }}$ & 0.4 & 0.6 \\
\hline $\mathrm{Pe}_{\text {low }}$ & 20 & 50 \\
\hline $\mathrm{Pe}_{\text {high }}$ & 50 & 82 \\
\hline$\square \mathrm{t}$ & 0 & 50 \\
\hline
\end{tabular}

\section{Improved Chaotic Particle Swarm Optimization (ICPSO) Algorithm}

Particle swarm optimization was presented by Kennedy and Eberhart at IEEE International Conference on Neural Networks in 1995 [8]. Particle swarm optimization is a biologically-based algorithm that can be applied to the domain of combinatorial 
optimization. PSO can dynamically adjust the velocity and trajectory of each particle in the solution space based on the flight experience of itself and others. The state of each particle can be described by a set of position and velocity vectors, which donate the feasible solutions and the direction of movement in the solution space respectively [9]. In one-iteration, each particle adjusts its own trajectory in order to move towards its best position and the global best according to the following equations:

$\left\{\begin{aligned} V_{i j}(t+1)= & w \times V_{i j}(t)+C_{1} \times r_{1} \times\left(\text { pbest }_{\mathrm{ij}}(\mathrm{t})-\mathrm{x}_{i j}(\mathrm{t})\right) \\ & +C_{2} \times r_{2} \times\left(\text { gbest }_{j}(\mathrm{t})-\mathrm{x}_{i j}(\mathrm{t})\right)\end{aligned}\right.$

for $\mathrm{j}=1, \ldots, \mathrm{d}$, where $\mathrm{d}$ is the number of dimensions; $\mathrm{i}=1, \ldots, \mathrm{n}$ where $\mathrm{n}$ is the number of particles; $\mathrm{t}$ is the iteration number, $w$ is the inertia weight, $r_{1}$ and $r_{2}$ are two random numbers uniformly distributed in the range $[0,1] ; c_{1}$ and $c_{2}$ are the acceleration factors.

\subsection{Chaotic Mapping}

In standard particle swarm optimization (SPSO), the initial population is randomly selected, and the solution space is too large to guarantee uniformity in the whole solution space traversal. Therefore, the global search ability of SPSO would be reduced, which makes the algorithm trapped in local optimal solution. The ergodicity of chaotic mapping can effectively overcome the above problems. Using the typical Logistic mapping to generate chaotic variables, and it can be turned into the optimization variables by linear mapping:

$$
x_{n+1}=4 x_{n}\left(1-x_{n}\right)
$$

$$
x \in(0,0.5) \cup(0.5,1)
$$

$$
y_{n}=a+(b-a) x_{n}
$$

Where $b$ and $a$ are the upper and lower limit of the optimization variables respectively. When the above conditions are met, the chaotic variables can completely traverse on the interval $[0,1]$, and corresponding optimization variables can traverse on its value range. So the ergodicity of initial population can be ensured in the whole solution space.

\subsection{Exponential Function Inertia Weight Adjustment Strategy}

In PSO, the inertia weight is an important adjustable parameter. The larger one can enhance the global search ability of the algorithm, and the smaller one can enhance the local search ability [10].

As shown in Ref.5, in comparison with linear function and quadratic function, exponential function inertia weight adjustment strategy can achieve higher accuracy and faster convergence speed in solving unimodal or multi-peak function optimization problem.

The equations of exponential function inertia weight adjustment strategy are as follows:

$$
\begin{gathered}
w=\left(w_{\text {end }}-\alpha\right)\left(\frac{w_{\text {sat }}}{w_{\text {end }}-\alpha}\right) 1 /\left(1+\beta \cdot(\mathrm{T}-1) /\left(\mathrm{T}_{\max }-1\right)\right) \\
\alpha=w_{\text {cad }}-\left(\frac{w_{\text {cud }}}{w_{\text {sat }}}\right) \frac{1}{\beta}
\end{gathered}
$$


Where $T_{\max }$ donates the maximum number of iterations; $T$ is the iteration number; $w_{\text {sart }}$ 、 $w_{\text {end }}$ are the initial and the final value of inertia weight respectively, which are specified as 0.9 and $0.4 ; \alpha, \beta$ are the adjustment coefficient of exponential function, which are generally specified as 0.312 and 10 .

\subsection{Local Chaos Substitution}

In SPSO, when the algorithm encountered the local optimal solution during the optimization process, all particles are likely to fall into the local solution, which makes the algorithm stagnant and unable to get rid of the local optimal state. In this paper, chaotic particles are introduced to improve the ability of escaping from local optimal and strengthen the local search capacity. Therefore, when the PSO trapped in local optimal solution, using chaotic iterative to replace the particles with poor fitness and increase the diversity of the population.

\section{Parameters Optimization based on ICPSO Algorithm}

\subsection{Optimization Objective}

The goal of the energy management control strategy of E-REV is to improve the vehicle fuel economy and increase the driving range as much as possible without reducing the vehicle dynamic performance in the extended-range mode. The parameters optimization based on ICPSO algorithm is as follows:

$\left\{\begin{array}{c}\operatorname{Min} \mathrm{J}(\mathrm{X})=[\mathrm{FC}(\mathrm{X}), \operatorname{SOC}(\mathrm{X})] \\ g_{i}(\mathrm{X}) \geq 0, j=1,2, \ldots, n\end{array}\right.$

(5)

Where $\mathrm{X}$ donates the parameter vector; $\mathrm{FC}(\mathrm{X})$ donates the comprehensive onehundred-kilometer fuel consumption of E-REV, which equals to the engine fuel consumption plus the equivalent battery fuel consumption; $\mathrm{SOC}(\mathrm{X})$ donates the deviation from target SOC value. $g_{\mathrm{i}}(\mathrm{X}), \mathrm{j}$ is a set of nonlinear inequality constraints, which represents the vehicle dynamic performance demand, such as maximum speed, acceleration time and the change of battery.

The comprehensive one-hundred-kilometer fuel consumption can be achieved by calculating with the equivalent fuel consumption. An equivalent fuel consumption method can be calculated using Eq.(6).

(6)

$$
V_{\text {fuel }}=\frac{E_{k} \cdot 3600}{D_{\text {fuel }} \cdot Q_{\text {fuel_low }} \cdot \eta_{\text {eng }} \cdot \eta_{\text {gen }}}
$$

Where, $V_{\text {fuel }}$ is the equivalent fuel consumption (L); $E_{k}$ is the battery energy consumption; $D_{\text {fuel }}$ is the fuel density, which is defined as $0.85 \mathrm{~g} / \mathrm{cm}^{3}$ for diesel; $Q_{\text {fuel_low }}$ is low calorific value of fuel combustion, which is defined as $43000 \mathrm{~J} / \mathrm{g}$ for diesel; $\eta_{\text {eng }}$ and $\eta_{g e n}$ are the average efficiency of engine and generator during one driving cycle respectively.

\subsection{Parameters Optimization}

Based on ICPSO algorithm, the concrete steps of parameters optimization for E-REV energy management control strategy are as follows: 
(1) Initial population with chaotic mapping, set the population size as $30, C_{1}=C_{2}=2$, $T_{\max }=1000$, the chaos search replace probability is 0.3 , and stagnation times and chaos search times are 50 and 30 respectively.

(2) Calculate the fitness of all particles, and save the global optimal solution and individual optimal position to gbest and pbest respectively.

(3) Update the velocity and position of each particle using Eq.(1)

(4) If the algorithm is stagnated, turn to Step5, otherwise Step6.

(5) Generate chaos particles, local search near the gbest with the set chaos search times, replace the poor fitness particles with probability of 0.3 , update velocity and position of the remained particles using Eq.(1)

(6) Update the velocity and position of each particle using Eq.(1), calculate the fitness and update the value of gbest and pbest

(7) If the algorithm reach the termination condition, then stops, and output the optimal solution, otherwise turn to Step2.

The flow chart of ICPSO algorithm is illustrated in Figure 3:

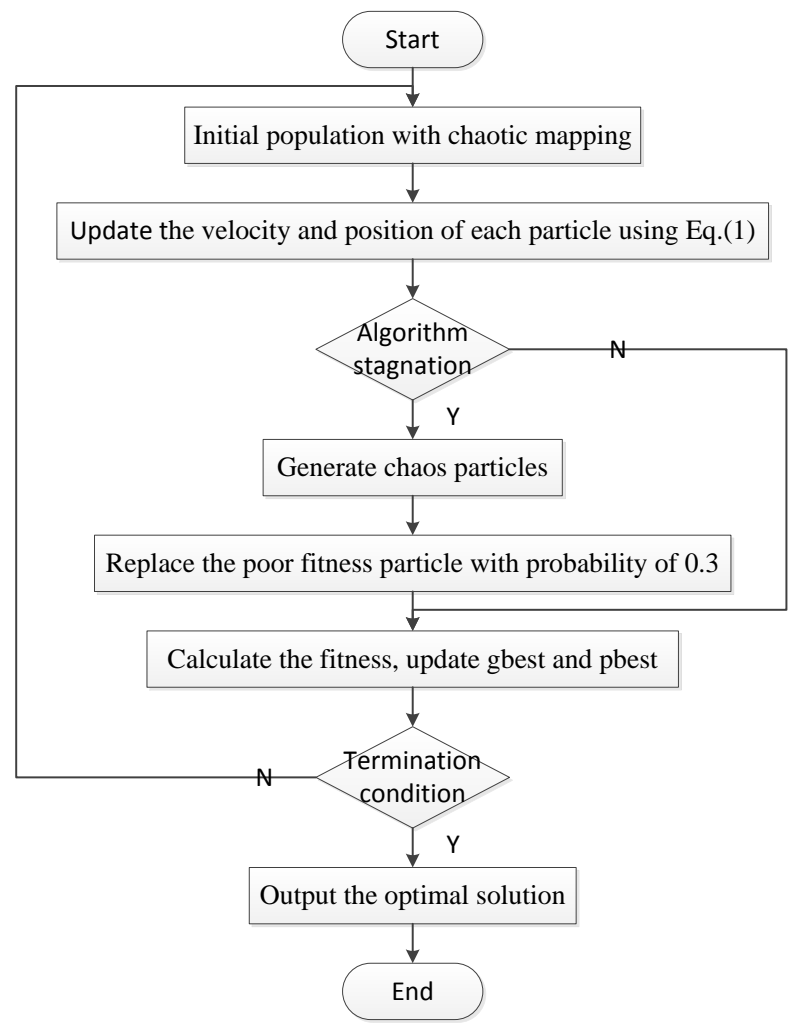

Figure 3. ICPSO Processes

\section{Simulation and Result Analysis}

In order to validate the accuracy and effectiveness of the proposed E-REV energy management control strategy, a backward-in-power nonlinear vehicle model is established in Matlab/Simulink which neglects the effects of driver. The China typical city bus cycle (CTCBC) is used as the basic driving cycle to the simulation based on GB/T 19754-2005. The initial value of SOC is set as 0.3 to reduce the program running time and obviously observe the change of SOC. The simulation results are shown in Figure 4- 5. 


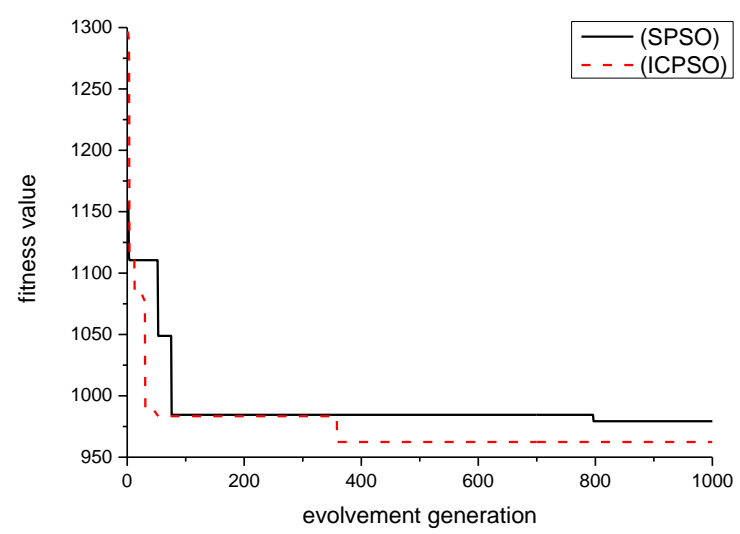

Figure 4. Convergence Rate Contrast Figure of SPSO and ICPSO

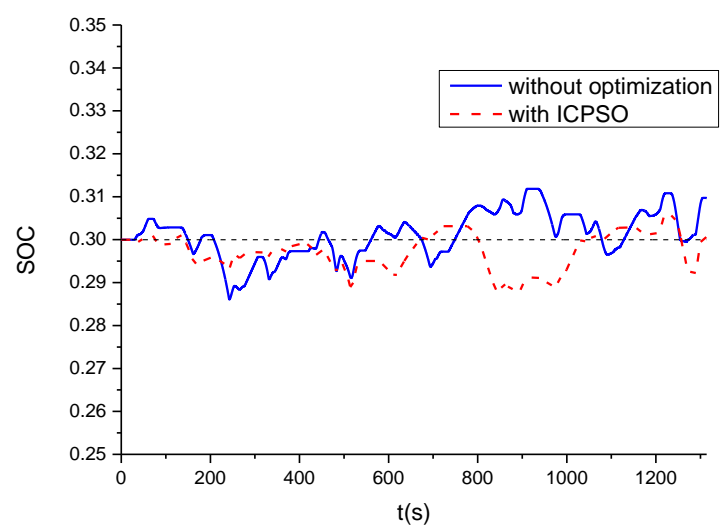

Figure 5. SOC Trajectories in One Driving Cycle

As shown in Figure 4, ICPSO can greatly improve convergence speed and optimizing precision than SPSO, this is due to the better local search capacity of it. If the algorithm was stagnated, ICPSO could escape from local optimal more quickly. Thus, ICPSO is better suits than SPSO in a multi-parameter optimization problem of E-REV energy management control strategy.

Figure 5 shows the battery SOC trajectories of optimal operation curve control strategy in one driving cycle. It shows that SOC can be limited at the range of 0.25 to 0.35 , and the final value is approximately equal to 0.3 , which proves that the proposed energy management control strategy is correct and effective. However, comparing to the nonoptimized one, parameters optimization with ICPSO can reduce the fluctuation of SOC, which means less effect on the battery during the process of driving.

The optimization results are shown in Tab.3. Where, $Q_{c}$ is the Comprehensive onehundred-kilometer fuel consumption, $\mathrm{Q}_{\mathrm{f}}$ is the Engine one-hundred-kilometer fuel consumption, $S O C_{\text {final }}$ is the final value of SOC. 
Table 3. Optimization Results

\begin{tabular}{c|c|c}
\hline Optimization variables & without optimization & ICPSO optimization \\
\hline$S O C_{\text {low }}$ & 0.3 & 0.3377 \\
\hline$S O C_{\text {hish }}$ & 0.5 & 0.4158 \\
\hline $\mathrm{Pe}_{\text {low }}$ & 35 & 39.6591 \\
\hline $\mathrm{Pe}_{\text {hish }}$ & 65 & 58.3421 \\
\hline$\square \mathrm{t}$ & 30 & 0.6384 \\
\hline $\mathrm{Q}_{\mathrm{c}}(\mathrm{L} / 100 \mathrm{~km})$ & 29.1253 & 26.4896 \\
\hline $\mathrm{Q}_{\mathrm{f}}(\mathrm{L} / 100 \mathrm{~km})$ & 34.1638 & 28.5294 \\
\hline$S O C_{\text {final }}$ & 0.3097 & 0.3006 \\
\hline
\end{tabular}

In general, the one-hundred-kilometer fuel consumption of a traditional diesel bus is $48 \mathrm{~L} / 100 \mathrm{~km}$ on average. And this of a traditional hybrid electric bus is $40 \mathrm{~L} / 100 \mathrm{~km} \mathrm{[11].}$ Therefore, in comparison with the other traditional vehicles of the same size, the optimal operation curve control strategy could observably improve the economic performance of E-REV. That is, the proposed strategy is very effective.

On the basis of the optimization results, parameters optimization with ICPSO can improve control performance. The $\mathrm{Q}_{\mathrm{c}}$ and $\mathrm{Q}_{\mathrm{f}}$ are reduced by $9.05 \%$ and $16.5 \%$ respectively, and the final value of SOC is closer to the target.

\section{Conclusions}

(1)The optimal operation curve control was provided to investigate the energy management strategy for E-REV. The full vehicle dynamic model and control strategy were built based on Matlab/Simulink, and the simulation was conducted under typical city driving cycles. The simulation results show that, the battery SOC can be limited to a small range, and APU works on the optimal operation curve, which means less fuel consumption, better economy and extending of driving range.

(2)Aiming at the shortcomings of SPSO, an improved chaotic particle swarm optimization algorithm was proposed to optimal the key parameters of E-REV energy management control strategy. The results indicate that ICPSO can greatly improve convergence speed and optimizing precision compared with SPSO, showing the superiority of ICPSO in respect of the effects of control parameter optimization for EREV.

(3)With ICPSO optimization, the comprehensive fuel consumption and engine fuel consumption of E-REV are reduced by $9.05 \%$ and $16.5 \%$. In addition, the battery SOC changes more gently and the final value of SOC is closer to the target, which could effectively control the variation of battery and benefit battery life.

\section{Acknowledgements}

This research was supported by the National Natural Science Foundation of China under Grants 51175043 and 51205022, International S\&T cooperation program of China (ISTCP) under Grant 2015DFG8190, National Sci-Tech Support Plan under Grants 2014BAG02B02 and 2015BAG02B00, Foundation of Engineering Research Center for Electric Vehicle of Beijing, and Foundation of Beijing Institute of Technology under Grant 20140342015. Any opinions, findings, and conclusions are those of the authors and do not necessarily reflect the views of the above agencies. 


\section{References}

[1] E. D. Tate, Michael O. Harpster and Peter J. Savagian. The Electrification of the Automobile: From Conventional Hybrid, to Plug-in Hybrids, to Extended -Range Electric Vehicles. 2008 World Congress Detroit, April 14-17; Michigan ( 2008)

[2] D. Feroldi, M. Serra and J. Riera. Energy management strategies based on efficiency map for fuel cell hybrid vehicles, Journal of Power Sources. Vol. 190, Issue 2, ( 2009), pp. 387-401.

[3] J. WU, C.-H. ZHANG, N.-X. CUI. PSO algorithm-based parameter optimization for both HEV powertrain and its control strategy, International Journal of Automotive Technology, Vol. 9, Issue 1, ( 2008) , pp. 53-69.

[4] W. Guangqiang, C. Huiyong. Multi-Objective Optimization of HEV Parameters Based on Genetic Algorithm, Automotive Engineering, Vol. 31, Issue 1, ( 2009), pp. 60-64 .

[5] L. Duyou. Simulation and Experimental Study on Ride Comfort Optimization of a Mine Car, Beijing, Beijing Institute of Technology, January (2015)

[6] Z. Su, N. Jigao, C. Fengxiang and P. Fenglai. A Study on Powertrain Design and Simulation for Rangeextended Electric Vehicle, Automotive Engineering, Vol. 33, Issue 11, (2011), pp. 924-929.

[7] R. Matthe, L. Turner, H. Mettlach: VOLTEC battery system for electric vehicle with extended range, 2011- 4-1.

[8] J. Kennedy, R. Eberhart. Particle swarm optimization, Proceedings of IEEE International Conference on Neural Networks. Vol. 4, Issue 2, ( 1995), pp. 1942-1948.

[9] Q. Feng, Particle swarm optimization algorithm and its industrial application, Science Press, Beijing (2013)

[10] S. Suresh, R. V. Babu, and H. J. Kim. No-Reference Image Quality Assessment Using Modified Extreme Learning Machine Classifier, Applied Soft Computing, Issue 9, (2009), pp. 541-552.

[11] Zhu Wuxi, Sun Liqing. Control Strategy Research for Extended-range Electric Bus, Automobile Technology, Issue 4, (2009), pp. 1-5.

\section{Authors}

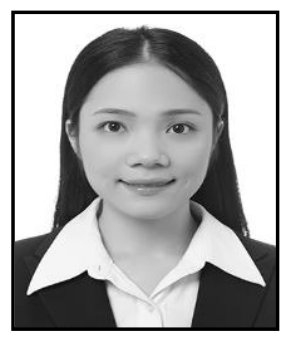

Yongchen Jiang, achieved her Bachelor degree in Vehicle Engineering from Wuhan University of Technology. She is a Graduate student of National Engineering Laboratory for Electric Vehicles, Beijing Institute of Technology. Her current research interest is electric vehicle control technology.

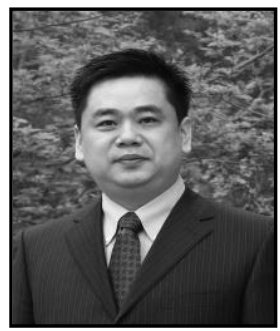

Cheng Lin.Professor and Ph.D supercisor of National Engineering Laboratory for Electric Vehicles, Beijing Institute of Technology, China. He has edited 4 professional publication and published more than 50 research papers in domestic and overseas academic journals. His research interests are vehicle electrification technology and electric vehicle integration theory.

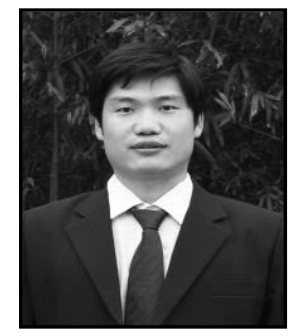

Wanke Cao, achieved his $\mathrm{Ph} . \mathrm{D}$ in Vehicle Engineering from Northeastern University, China, in 2008. He currently working as a lecturer of National Engineering Laboratory for Electric Vehicles, Beijing Institute of Technology. His research interests are electric vehicle control technology and vehicle network control technology. 
International Journal of Grid and Distributed Computing Vol. 9, No. 9 (2016) 